\title{
LOS VIAJES DE LAS PERSONAS RESIDENTES EN CANARIAS: TURISMO Y MOVILIDADES
}

\author{
Manuel Ángel Santana Turégano* \\ Universidad de La Laguna
}

\section{RESUMEN}

Este trabajo analiza el turismo de las personas residentes en Canarias dentro del archipiélago, tanto en la propia isla como en otras, centrándose en las pernoctaciones en establecimientos turísticos registrados, haciendo uso de datos secundarios y desde la perspectiva del paradigma de las movilidades. El análisis realizado sugiere que existen diferencias importantes en la propensión a consumir servicios de alojamiento en las islas entre las dos islas más pobladas, lo que se interpreta en clave de condicionantes tanto culturales como de comercialización. El escaso uso de la información disponible sobre el transporte, y las dificultades para conceptualizar algunos de los fenómenos analizados, como las pernoctaciones en la propia isla, apoyan la idea de que sería beneficioso adoptar el paradigma de las movilidades para comprender mejor los fenómenos tradicionalmente asociados en Canarias al turismo. Palabras Clave: Canarias, transporte, turismo interior, movilidades, turismo fordista.

\section{TRAVELLING OF PEOPLE LIVING AT THE CANARY ISLANDS: TOURISM AND MOBILITY}

\section{Abstract}

This paper analyzes domestic tourism in the Canary Islands, using secondary data and following an approach based in the mobilities paradigm, using the night stays as the main indicator. The analysis shows that the two most populated islands differ in their propensity to consume lodging services, and these differences are considered to be due to both cultural and commercialization reasons. Due to the scarce utilization of statistics already available, and the difficulties that stem from trying to analyze phenomena such as night individuals spent in tourism facilities in the same island where they live, it seems that adopting an approach based in the mobilities paradigm would be beneficial for better understanding phenomena traditionally associated in the Canary Islands with tourism.

KEYwORds: Canary Islands, transportation, domestic tourism, mobilities, fordist tourism. 


\section{INTRODUCCIÓN}

¿Qué es el turismo? La definición habitual podría sintetizarse en torno a la idea de que el turismo son "las actividades que realizan las personas durante sus viajes y estancias en lugares distintos a los de su entorno habitual, por un período inferior a un año, con fines de ocio, por negocio y otros motivos» (Álvarez Sousa et al., 2019: 7; OMT/WTO, 2018). Sin embargo, desde hace casi ya dos décadas el paradigma de las movilidades (Sheller \& Urry, 2006; Urry, 2012; Urry, 2016) plantea que es necesario "pensar y diluir el turismo en el contexto internacional de los procesos de movilidad y de creciente circulación de personas y mercancías» (Álvarez Sousa et al., 2019: 18). Y es que algunos de los conceptos que tradicionalmente tienden a darse por supuestos en el paradigma del turismo, como la clara diferenciación entre un «entorno habitual» desde el que se sale y al que se regresa, implícito en la idea de "tour» asociada al turismo, tiene ya muy poca utilidad en las sociedades globalizadas e hiperconectadas del siglo Xxi. Aun, es más, la pandemia de la covid-19, que durante el año 2020 cambió por completo las pautas de movilidad a nivel mundial, previsiblemente va a seguir afectando a estas.

Dentro del estudio del turismo desde las Ciencias Sociales la Sociología del Turismo se concebía tradicionalmente como «una especialidad emergente que se ocupa del estudio de las motivaciones turísticas, los roles, las relaciones y las instituciones turísticas, así como las repercusiones sobre los turistas y sobre las sociedades que los acogen» (Cohen, citado en Álvarez Souza et al. 2019: 15). Esta conceptualización de la Sociología del Turismo es deudora de una división del trabajo intelectual en las Ciencias Sociales según la cual la Sociología se encargaría de los valores (qué lleva a la gente hacer turismo, cuáles son sus motivaciones), mientras que la Economía se encargaría del valor, de la creación del valor, y que derivaría del pacto realizado por Talcott Parsons con los economistas de la Universidad de Harvard en la década de 1940 (Camic, 1987; Stark, 2009). Sin embargo, a medida que a partir de la década de 1980 este pacto empieza a romperse surgen aproximaciones distintas desde las Ciencias Sociales al turismo. Así, la economía política (Political Economy) del turismo podría definirse como «el estudio de las fuerzas socioeconómicas y de las relaciones de poder que se constituyen en el proceso de producción de mercancías para el mercado y de las divisiones, conflictos y desigualdades que surgen» (Bianchi, 2017; Bianchi, 2018: 89 traducción y adaptación propia). En cualquier caso, y siguiendo el viejo adagio sociológico de que «las definiciones de la realidad crean realidad», por más problemática que sea la definición del turismo que realiza la Organización Mundial del Turismo (OMT), fundada en 1975 y dependiente del Consejo Económico y Social de las Naciones Unidas, ha servido para el fin para el que se ideó: «crear un marco conceptual básico capaz de ordenar (al menos en alguna medida) la recopilación y análisis de estadísticas» (Álvarez Souza et al. 2019). En

*E-mail: masantur@ull.es. Universidad de La Laguna. 
un muy buen ejemplo de la performatividad de las disciplinas económicas (Callon, 2010), la forma en que se recogen las estadísticas ha ayudado a prestar importancia a unos fenómenos y restarla a otros. Así, pese a que es sabido que es mucho más importante el turismo interno o doméstico que el internacional, el foco se ha tendido a poner en este último. Y pese a que la información disponible señala que al menos la mitad de los viajes que podrían considerarse "turísticos» según la definición habitual se realiza utilizando alojamientos de no mercado (se viaja para visitar amigos y familiares), se tiende a asociar el turismo a la hostelería. Cuando lo cierto es que quienes viajan para visitar a amigos y familiares y se alojan en sus viviendas realizan turismo, aunque no pernocten en establecimientos hoteleros.

La recogida de datos para generar conocimiento sobre el turismo se ha realizado asumiendo, de manera implícita, el reparto del trabajo intelectual entre Sociología y Economía aludido anteriormente. Así, el conocimiento de las motivaciones que llevan a la gente a practicar turismo se ha hecho a través de «encuestas de turismo emisor", y este sentido España ha sido un país pionero, realizando desde 1996 la encuesta FAMILITUR, que estudia el comportamiento turístico de los españoles (Aranda Palmero, 2001) ${ }^{1}$. Este tipo de investigaciones han permitido obtener conocimiento acerca de cuestiones como la propensión a viajar de los habitantes de un determinado lugar, el número de viajes que se realizan y sus motivaciones. Permiten saber, por ejemplo, que en España cerca del 60\% de la población practica turismo, que los viajes suelen tener motivos relacionados con el ocio y que en más de la mitad de las pernoctaciones se usan alojamientos de no mercado, como pueden ser segundas residencias, o casas de amigos o familiares. Estos datos evidencian las limitaciones de los enfoques tradicionales sobre el turismo, que tienden a hacerlo sinónimo de «industria hotelera». Dado que menos de la mitad del turismo que realizan los espańoles se puede asociar a establecimientos turísticos, el paradigma de las movilidades puede resultar más útil para comprender los fenómenos asociados de informalización económica de la actividad inmobiliaria y sus imprecisas fronteras con el turismo. Estos datos también permiten realizar comparaciones entre distintos estados y regiones o entidades subnacionales (para un ejemplo de este tipo de estudios puede verse, por ejemplo, Rodríguez González \& Santana Turégano, 2014). Y también se han utilizado para estimar, de forma indirecta, el impacto económico de lo que se suele denominar «sector turístico». Sin embargo, basta ver la metodología ${ }^{2}$ que suele aplicarse en este tipo de estudios para entender las limitaciones que en la actualidad implica el "paradigma del turismo»: en la

1 Desde 2015, al pasar a realizarse por el Instituto Nacional de Estadística (INE) se ha transformado en la «Encuesta de Turismo de Residentes».

${ }^{2}$ Así, tomando como ejemplo el cuestionario de la Encuesta de Turismo de Residentes que realiza el INE se pregunta a los encuestados por los viajes realizados, que han implicado al menos una pernoctación, a municipios distintos al de residencia, y luego se pide a la persona entrevistada que indique cuál ha sido el motivo principal, entendiendo como tal aquel sin el cual no habría tenido el viaje, y se les da para elegir entre una lista de motivos en los que se incluye, por ejemplo, el «turismo de sol y playa», «turismo cultural» o «turismo de naturaleza», entre otros. Puede consultarse el cues- 
medida en que el turismo se acaba definiendo en estos estudios por su motivación "distinta al negocio» y por realizarse en entornos "distintos a la residencia habitual», lo que a menudo puede acabar sucediendo es que se presenta, bajo el supuesto carácter de «objetividad» que se atribuye a las estadísticas, un conjunto heterogéneo de datos que no se sabe muy bien qué significan, y que a menudo ponen en el mismo cajón realidades muy diversas. Un «entorno distinto a la residencia habitual» puede ser tanto un municipio situado a 50 kilómetros de distancia como un país lejano y de cultura distinta. Afirmaciones del tipo «el $70 \%$ de los españoles buscan turismo de sol y playa, y el $45 \%$ de los daneses turismo deportivo" son en realidad generalidades que poco aportan, pues podría considerarse «turismo de sol y playa» tanto una luna de miel de una pareja de madrileños en Mauricio como las vacaciones de unos grancanarios en el sur de su propia isla; y "turismo deportivo de los daneses» puede ser tanto un viaje de un par de días en bicicleta por las afueras de Copenhague como un viaje de trekking al Himalaya.

El otro gran corpus de investigaciones que se han realizado sobre el turismo es el que abarca todo el abanico de las distintas "Encuestas del Gasto Turístico», que se llevan realizando desde hace bastante tiempo en los principales destinos. $\mathrm{Si}$ desde el punto de vista del turismo emisor el turismo alemán, británico, español o canario, por ejemplo, haría referencia al consumo turístico que hacen las personas residentes en Alemania, Gran Bretaña, España o Canarias, independientemente de dónde vayan, el enfoque aquí es distinto: las encuestas en destino entienden que el «turismo de Canarias», por ejemplo, sería la suma de todos los consumos turísticos que se realizan en Canarias. La idea de estos estudios es conocer, por un lado, las características sociodemográficas de los turistas que visitan un determinado destino: de qué lugares proceden, qué edades, profesiones, niveles de estudio y de ingresos poseen, en qué tipo de grupos viajan. Pero a menudo el objetivo principal de este tipo de estudio es fundamentalmente conocer cuánto y en qué gastan el dinero los turistas. Y el objetivo último acaba siendo, a menudo, el de estimar el dinero que aporta el turismo a una determinada economía, y por ello han acabado desarrollándose metodologías como la Cuenta Satélite del Turismo, bajo los auspicios de la OMT. Como ejemplo del tipo de información que se produce desde este ámbito, el Instituto de Estadística de Canarias publica periódicamente no solo los ingresos por habitación, sino los ingresos totales generados por el turismo. Con lo cual, como podemos ver, y aunque no se diga explícitamente, al final el estudio del turismo se acaba centrando en el de los viajes que generan ingresos a la industria hotelera y prestando menos atención, por ejemplo, a los viajes a segundas residencias o aquellos en que se va a visitar a amigos y familiares y se utilizan sus domicilios como alojamiento.

Siguiendo recomendaciones de la OMT, la información estadística sobre el turismo, sector denominado a veces "Hostelería y Turismo», se suele dividir en

tionario en https://www.ine.es/daco/daco42/etr/etr_cuestionario.pdf y la metodología puede encontrarse en https://www.ine.es/daco/daco42/etr/etr_metodologia.pdf. 
dos grandes áreas, por un lado "Demanda turística: turistas y pasajeros» y por otro «Oferta Turística: Hostelería, Restauración y otros ${ }^{3}$ ». La propia terminología utilizada da por sentado que en turismo siempre hay "oferta" o "demanda», dado que se asume que el turismo es un fenómeno mercantilizado. En la medida en que el turismo, al final, se acaba haciendo en la práctica sinónimo de las industria hostelera y del transporte, una importante fuente de información estadística sobre el «sector turístico» es la que se genera a partir de estas empresas. En el caso español, a partir de la década de 1960, cuando se dan a la vez el crecimiento del sector turístico y la modernización tecnocrática de la economía, el Instituto Nacional de Estadística (INE) comienza a publicar los resultados de la Encuesta de Movimientos de Viajeros en Establecimientos Hoteleros y en Acampamentos Turísticos, que a partir de 1999 se complementan con la Encuesta de Ocupación en Apartamentos Turísticos, a las que se añaden en 2001 los establecimientos de turismo rural y en 2014 los albergues turísticos (González Martín \& Velasco Gimeno, María de Carmen, 2021). Siguiendo la lógica de las empresas industriales, de las cuales se recababan datos sobre la producción, las distintas Encuestas de Ocupación Hotelera (nombre genérico bajo el que se agrupan todas) ofrecen información tanto sobre el número de viajeros alojados como sobre las pernoctaciones que estos realizan, siendo este último el indicador más fiable de la producción de la industria alojativa ${ }^{4}$.

En los últimos ańos el desarrollo de los sistemas de telecomunicaciones y de lo que se ha dado en llamar Big Data ha acabado generando una importante revolución en las estadísticas sobre el turismo, ya que al haberse automatizado las encuestas de alojamiento han adquirido un carácter censal. En el caso de Canarias, el ISTAC recoge datos, para todos los establecimientos turísticos registrados, de los viajeros alojados y de las pernoctaciones realizadas, así como de los precios, lo que permite elaborar, entre otros indicadores, los índices de ocupación, los ingresos por habitación disponible (RevPar, Revenue per Available Room) y la tarifa media (ADR, Average Daily Rate). Todo este desarrollo tecnológico ha llevado a que un mismo tipo de dato pueda recabarse al menos de dos maneras distintas, que presentan distintos niveles de fiabilidad. Si, por ejemplo, quisiéramos saber las nacionalidades más frecuentes entre los turistas que visitan un determinado destino turístico (pongamos por caso la isla de Lanzarote) la forma de estimarlo desde las estadísticas de la oferta sería sumando los viajeros alojados y las pernoctaciones en los hoteles y apartamen-

${ }^{3}$ Los nombres corresponden a los que utiliza el Instituto de Estadística de Canarias (ISTAC), véase http://www.gobiernodecanarias.org/istac/estadisticas/sectorservicios/hosteleriayturismo/. Para el caso de España, el INE, que ofrece las estadísticas sobre turismo en el apartado «Servicios», no hace una diferenciación tan clara, pero también ofrece estadísticas sobre los españoles como demandantes de servicios turísticos (la ya referida «Encuesta de Turismo de Residentes» y también Frontur) y sobre la oferta turística en Espańa: Encuesta de Ocupación Hotelera, de Apartamentos, de Albergues y Cámpines. Las estadísticas de transporte en el INE están separadas de las de Turismo.

${ }^{4} \mathrm{Si}$ una persona se aloja en un hotel durante 7 noches se contaría un único viajero y 7 pernoctaciones; si en otro hotel, una misma habitación es ocupada durante 7 noches consecutivas por siete personas distintas se contabilizarían 7 pernoctaciones y 7 viajeros. 
tos legalmente registrados. Esta manera de recabar los datos tiene el inconveniente de que deja fuera de la contabilización a quienes se alojan en establecimientos no registrados (viviendas vacacionales y/o establecimientos ilegales). Por el contrario, desde el punto de vista de la demanda podríamos preguntar a los turistas en qué hotel, zona o municipio se han alojado, siendo el principal inconveniente de esta forma de recabar los datos las imprecisiones y confusiones por parte de los clientes 5 .

Y por último, podríamos estimar las nacionalidades más frecuentes entre los turistas que visitan Lanzarote, siguiendo con el ejemplo, a través de la nacionalidad de los pasajeros o del origen de los vuelos, lo que implicaría el problema de discriminar entre turistas y residentes. $\mathrm{Y}$ es que, si queremos saber a dónde viajan los españoles, además de preguntarlo, como se hace en la Encuesta de Turismo de Residentes, podemos obtener esos datos a partir de las estadísticas de transporte, y la precisión del indicador utilizado dependerá de diversas variables. En territorios como Canarias, medir el origen de los turistas a partir del origen de los vuelos es un método relativamente exacto, pues si tenemos en cuenta que esto nos permite saber el origen también de quienes vienen de otras islas, si estimamos el origen de los turistas en función de las conexiones marítimas y aéreas solo dejaríamos de contabilizar el turismo que se da en el interior de cada isla (cosa que, en cualquier caso, podemos contabilizar en las estadísticas de alojamientos). Por último, y aunque en ese ámbito el desarrollo es aún más limitado, la extensión de sistemas de pago mediante tarjetas y el progreso en el tratamiento de los datos hacen posible que en la actualidad, muchas de las cuestiones que se solían incluir en las encuestas sobre el gasto turístico acerca de cuánto y en qué gastan su dinero los «turistas» podrían estimarse mediante este tipo de mecanismos.

El objetivo de este trabajo es acercarse, desde el paradigma de las movilidades, al conocimiento del turismo doméstico que realizan las personas residentes en Canarias en el interior del archipiélago. Para ello, en primer lugar, se contextualizará este tanto en el conjunto de las movilidades que se realizan en las islas como en el conjunto del turismo que se desarrolla en Canarias, tanto por parte de las personas que residen en las islas como de las que provienen del exterior. El análisis se centrará en las personas que se alojan en establecimientos turísticos registrados, por lo que se utilizarán las distintas encuestas existentes. Por ello, en realidad lo que acabaremos analizando es el consumo por parte de la población local de servicios elaborados por la industria turístico-alojativa. Ello sugiere la necesidad, como veremos posteriormente, de imbricar los análisis empíricos con los debates teóricos sobre la diferenciación del turismo y otras formas de ocio (Santana Turégano, 2000). Por ello acabaremos planteando, enlazando con el paradigma de las movilidades, hasta qué punto es conveniente revisar algunos de los conceptos habitualmente utilizados, como el propio término de "turista», y no sería conveniente sustituirlos por otros conceptualmente menos cargados.

Los clientes no siempre recuerdan el nombre de su hotel/apartamento; a menudo confunden dónde está ubicado, generalmente no saben el municipio al que pertenece. 


\section{TURISMO Y MOVILIDADES EN CANARIAS}

Con entre 13 y 15 millones de turistas y más de 100 millones de pernoctaciones anuales Canarias se ha consolidado, en la segunda década del siglo XXI, como la región europea líder en turismo receptivo (ISTAC, 2021c; Kotzeva et al., 2018; Kotzeva et al., 2019). Esta extraordinaria importancia del turismo en Canarias ha producido innumerables estudios sobre las islas como destino, pero hasta la fecha no se han realizado investigaciones acerca de las islas como emisoras de turistas. Canarias es un caso especialmente interesante para el estudio de las movilidades, y para comprobar hasta qué punto el paradigma del turismo sigue siendo útil o si sería más productivo un enfoque basado en el paradigma de las movilidades, hasta el punto de que ha llegado a ser considerado un «laboratorio del turismo» (Díaz et al., 2017). Debido al carácter insular y a la lejanía, la práctica totalidad de los pasajeros (turistas o no) que llegan a las islas lo hacen por vía aérea, lo que facilita la producción de estadísticas ${ }^{6}$. Por otro lado, si recordamos que uno de los problemas de la definición habitual del turismo es que esta se hace en términos de viaje fuera del «entorno habitual», lo que es un término bastante impreciso, el carácter insular podría ayudar a operacionalizar este concepto, entendiendo a la propia isla de residencia como tal. Por último, debido a la importancia de la población residente nacida fuera del archipiélago ${ }^{7}$ es más que razonable pensar que existe un importante movimiento de personas que seguramente se comprende mejor desde el paradigma de las movilidades que desde el del turismo. Así, las personas que provienen del resto de España o del extranjero pero residen en Canarias es posible que vuelen a sus lugares de origen, pero como turistas, y que gente de sus lugares de origen vaya a visitarlos, dentro de lo conocido como turismo VFR (Visiting Friends and Relatives).

Cuando se habla de turismo en Canarias se tiende a pensar en las personas que vienen del extranjero y del resto de España a pasar unos días en Canarias, si bien, en puridad, se debería hablar del consumo de servicios y actividades típicamente turísticas en las islas, independientemente del lugar de origen de las personas que lo realizan. En realidad el turismo en Canarias incluye, además del turismo de los extranjeros y del de los españoles del resto de comunidades autónomas en Canarias, que es en lo que habitualmente se suele pensar, también al turismo que realizan las personas residentes en Canarias dentro del archipiélago ${ }^{8}$, tanto cuando las personas de una isla se desplazan a otras como cuando realizan turismo dentro

${ }^{6}$ Puede ser difícil estimar cuántos turistas llegan en el mes de agosto a Salou o Benidorm, pero en el caso de Canarias, y pese a que es necesario diferenciar entre pasajeros y turistas, las estadísticas de pasajeros por vía aérea constituyen un marco de referencia muy válido.

El extraordinario crecimiento demográfico de las islas en los últimos años ha ocasionado que, de acuerdo a los datos de la explotación estadística del Padrón para 2020, el 20\% de la población de las islas había nacido en el extranjero, y casi otro $10 \%$ en el resto de España, y que en Fuerteventura y Lanzarote la población nacida fuera de Canarias es tanta o más que la nacida en el archipiélago.

${ }^{8}$ Quedaría fuera por tanto el turismo que realizan las personas residentes en Canarias cuando viajan al exterior. 
de la propia isla. Y este último fenómeno, que, como veremos posteriormente, es bastante más importante de lo que se suele pensar, es a menudo invisibilizado por la imprecisa conceptualización de lo que se entiende por turismo. Si entendemos el turismo como «desplazarse fuera del entorno habitual» a veces cuesta pensar, dada la extensión de las islas, que se pueda salir del entorno habitual dentro de la propia isla ${ }^{9}$ y por lo tanto practicar turismo, pero si entendemos el turismo como «realización de consumo turístico» comprenderemos mejor por qué, en determinados entornos y épocas del año, buena parte de los «turistas» que encontramos residen en la misma isla que analizamos.

Con todas las matizaciones anteriormente señaladas, cuando se habla de «el turismo en Canarias» se suele pensar, en términos numéricos, en más de 13 millones de turistas extranjeros y 100 millones de pernoctaciones anuales, en una oferta alojativa de casi 400000 plazas, en más de 60000 empleos directos en la oferta alojativa y en una aportación al PIB que puede cifrarse en algo más de un tercio (Hernández Martín, 2011). De acuerdo a estas cifras, puede estimarse que en términos globales el turismo en Canarias se reparte entre un 35\% en Tenerife, un 30\% en Gran Canaria, un 18\% en Lanzarote, un 15\% en Fuerteventura y el 2\% restante en las denominadas «islas verdes ${ }^{10}$. Estas cifras incluyen tanto al turismo que realizan los residentes en Canarias como el de las personas que vienen desde fuera, y no ponen el movimiento turístico en el marco más amplio del movimiento de personas en Canarias, es decir, de las personas que, como quiera que las consideremos, llegan o salen de las islas por vía marítima o aérea. A partir de la recopilación de estadísticas de transporte aéreo y marítimo, que ofrece el Instituto de Estadística de Canarias (ISTAC 2021d), y tomando como referencia el año 2019 (las cifras de 2020 resultan muy alteradas por la pandemia de covid-19) podría estimarse que en Canarias entran al año más de 33 millones de pasajeros ${ }^{11}$, que corresponden a los aproximadamente 13 millones de turistas provenientes del extranjero, a unos 2 millones de turistas provenientes del resto de España y unos cerca de 1,7 millones de turistas de Canarias. El resto, es decir, unos 16 millones de pasajeros, podrían considerarse excursionistas, es decir, personas que se desplazan o bien de una isla a otra (la inmensa mayoría) o bien desde el exterior hasta alguna de las islas canarias

9 Si bien la extraordinaria variedad climática y paisajística del archipiélago hace que, dentro de una misma isla, se pueda vivir en entornos completamente distintos, de lugares húmedos y verdes a lugares secos y áridos.

${ }^{10} \mathrm{La}$ Palma, La Gomera y El Hierro; este reparto, con escasas variaciones, se obtiene tanto si se contabilizan turistas como si se cuentan pernoctaciones, establecimientos o plazas alojativas.

${ }^{11}$ En los 8 aeropuertos insulares, en los puertos del Estado en Canarias, que corresponden a Arrecife, Puerto del Rosario y Puerto de la Luz y de Las Palmas para la autoridad portuaria «Puertos de Las Palmas» y Santa Cruz de Tenerife, Santa Cruz de La Palma, Los Cristianos, San Sebastián de La Gomera, Santa Cruz de La Palma para la autoridad portuaria «Puertos de Tenerife», y en los puertos dependientes de la entidad «Puertos Canarios», en los cuales el grueso del tráfico de pasajeros se da en los de Órzola y Playa Blanca en Lanzarote, Corralejo y Morro Jable en Fuerteventura, Agaete en Gran Canaria y Vueltas en La Gomera. 
y vuelven a su casa en el mismo día, sin pernoctar ${ }^{12}$. De todos estos pasajeros podría estimarse que el $65 \%$ se mueven por vía aérea, y el resto por vía marítima. Casi la mitad de los pasajeros (47\%) que llegan a una isla canaria provienen de otra isla, ya sea por vía aérea o marítima ${ }^{13}$, cerca de un $13 \%$ llegan del resto de Espańa y en torno al $40 \%$ del extranjero. A partir de la recopilación de las estadísticas de transporte aéreo y marítimo en Canarias puede realizarse una aproximación al movimiento de personas entre las distintas islas canarias.

\begin{tabular}{|c|c|c|c|c|c|c|}
\hline \multicolumn{7}{|c|}{$\begin{array}{l}\text { CUADRO 1. MOVIMIENTO DE PASAJEROS EN PUERTOS } \\
\text { Y AEROPUERTOS CANARIOS POR ISLAS Y ORIGEN, } 2019\end{array}$} \\
\hline \multicolumn{3}{|c|}{ Vía marítima } & \multicolumn{3}{|c|}{ Vía aérea } & \multirow{2}{*}{ TотAL } \\
\hline IsLA & P. Canarios & P. Estado & Extranjero & Resto España & Resto Canarias & \\
\hline Lanzarote & 1550255 & 196371 & 2488512 & 535944 & 584807 & 5355889 \\
\hline Fuerteventura & 1810906 & 39757 & 1949754 & 350107 & 467551 & 4618075 \\
\hline Gran Canaria & 1183869 & 1278255 & 3611315 & 1537638 & 1370811 & 8981888 \\
\hline Tenerife & & 3635298 & 5029104 & 1975634 & 1358981 & 11999017 \\
\hline La Gomera & 119857 & 1391116 & & & 30746 & 1541719 \\
\hline La Palma & & 269173 & 176891 & 82499 & 441702 & 970265 \\
\hline El Hierro & & 166407 & & & 122591 & 288998 \\
\hline Canarias & 4664887 & 6976377 & 13255576 & 4481822 & 4377189 & 33755851 \\
\hline
\end{tabular}

Fuente: elaboración propia a partir de la Recopilación de Datos de Transporte Aéreo y Marítimo del ISTAC.

Por vía área, para el ańo 2019, se movieron en vuelos interinsulares un total de 4,3 millones de personas (es decir, casi 12000 pasajeros/día). Los flujos de personas más importantes fueron entre Tenerife y Gran Canaria, que con casi un millón de pasajeros anuales concentró casi el $23 \%$ del movimiento de personas por vía aérea en Canarias. De media unas 1370 personas llegaron cada día a Tenerife desde Gran Canaria (y viceversa) por vía aérea. Les siguen por orden de importancia el flujo entre Gran Canaria y Lanzarote, con unas 1100 personas por sentido cada día (más de 800000 pasajeros a lo largo de año), Tenerife y La Palma (1040 y 750000 , respectivamente), Gran Canaria y Fuerteventura (911 y 600 000), Tenerife y Lan-

${ }^{12}$ Es importante señalar que aquí se incluiría, por ejemplo, a turistas que se alojan en una isla y realizan una excursión de un día a otra.

${ }^{13}$ En La Gomera y El Hierro, que no tienen conexiones directas ni con el resto de Espańa ni con el extranjero, se llega, obviamente, al 100\%, y en La Palma, que posee pocas conexiones aéreas, al 73\%. Pero es interesante destacar el porcentaje del resto de islas: Lanzarote, 43,5\%; Fuerteventura, 50,2\%; Gran Canaria, $42,7 \%$ y Tenerife $41,6 \%$. Es decir, que, por ejemplo, a cada isla llegan casi tantas personas del resto de islas, por vía aérea o marítima, como del resto de Espańa y del extranjero (en este caso, por lejanía, básicamente por vía aérea), ya sean canarios que llegan a hacer excursionismo turismo y/o gestiones a la isla, o bien turistas que se alojan en otras islas y que durante un día hacen excursionismo en la isla en cuestión. 
zarote (539 pasajeros de media diaria, unos 390000 anuales) y Tenerife y Fuerteventura (407 diarios y 297000 anuales). En cuanto a las estadísticas del transporte marítimo, su explotación es algo más complicada, ya que no están desglosadas por origen y destino. En cualquier caso, y a título de mera ilustración, en el puerto de las Nieves (Agaete), que solo recibe pasajeros desde Tenerife, llegaron, para el año 2019, un total de 1183869 pasajeros, es decir, el equivalente a una media de 3243 personas diarias. Casi la misma cantidad de pasajeros (1 103601) se recibieron en Playa Blanca (Lanzarote), provenientes desde Corralejo (Fuerteventura).

A partir de la estimación de que la práctica totalidad de pasajeros llegados por vía marítima proceden del resto de las islas ${ }^{14}$, sumando los pasajeros llegados por mar al tráfico aéreo interinsular el siguiente cuadro muestra el origen de los pasajeros que llegan cada día a cada una de la islas para tres grandes orígenes: resto de Canarias, resto de España y extranjero:

\begin{tabular}{|c|c|c|c|c|c|c|c|}
\hline \multicolumn{8}{|c|}{ CUADRO 2. MOVIMIENTO DIARIO DE PASAJEROS EN CANARIAS* } \\
\hline \multicolumn{8}{|c|}{ ORIGEN } \\
\hline & \multicolumn{2}{|c|}{ Resto Canarias } & \multicolumn{2}{|c|}{ Resto España } & \multicolumn{2}{|c|}{ Extranjero } & \multirow[b]{2}{*}{ TOTAL } \\
\hline IsLa DESTINO & Авsoluto & $\%$ & Авsoluto & $\%$ & Авsoluto & $\%$ & \\
\hline Lanzarote & 6387 & $43,5 \%$ & 1468 & $10,0 \%$ & 6818 & $46,5 \%$ & 14674 \\
\hline Fuerteventura & 6351 & $50,2 \%$ & 959 & $7,6 \%$ & 5342 & $42,2 \%$ & 12652 \\
\hline Gran Canaria & 10501 & $42,7 \%$ & 4213 & $17,1 \%$ & 9894 & $40,2 \%$ & 24608 \\
\hline Tenerife & 13683 & $41,6 \%$ & 5413 & $16,5 \%$ & 13778 & $41,9 \%$ & 32874 \\
\hline La Gomera & 4224 & $100,0 \%$ & 0 & $0,0 \%$ & 0 & $0,0 \%$ & 4224 \\
\hline La Palma & 1948 & $73,3 \%$ & 226 & $8,5 \%$ & 485 & $18,2 \%$ & 2658 \\
\hline El Hierro & 792 & $100,0 \%$ & 0 & $0,0 \%$ & 0 & $0,0 \%$ & 792 \\
\hline Canarias & 43886 & $47,5 \%$ & 12279 & $13,3 \%$ & 36317 & $39,3 \%$ & 92482 \\
\hline
\end{tabular}

Fuente: elaboración propia a partir de la Recopilación de Datos de Transporte Aéreo y Marítimo del ISTAC**.

* Para esta tabla se ha dividido el total de pasajeros llegados por todas las vías en el año 2019 por los 365 días del año.

** Es importante recordar que las islas de La Gomera y El Hierro carecen de conexiones directas con el exterior, por lo que, aunque llegan a ellas cada día personas del resto de Espańa y del extranjero, lo hacen por vía marítima o área, fundamentalmente a través de Tenerife.

Resulta llamativo cómo, en un lugar como Canarias, en el que tiende a pensarse que el movimiento de pasajeros proviene fundamentalmente del exterior, para cada isla entre el 40 y el 50\% de los pasajeros que llegan cada isla provienen del resto del archipiélago. El movimiento de personas entre islas más importante, en términos cuantitativos, es el que tiene lugar entre Gran Canaria y Tenerife. En total, si a los pasajeros llegados a Gran Canaria desde Tenerife al puerto de Agaete

14 Solo un par de veces en semana llegan algunos pasajeros a los cuatro puertos (Arrecife, Santa Cruz de la Palma, La Luz y de Las Palmas y Santa Cruz de Tenerife) que tienen conexión con el puerto de Huelva. 
se suman los llegados al puerto de la Luz, y los llegados por vía aérea en total podría estimarse, para el año 2019, que una media de cerca de 6000 personas llegaron cada día a Gran Canaria desde Tenerife, y cabe pensar y lo mismo en sentido inverso, es decir, algo menos del 1\% de la población de la isla. Mucho más importante en términos relativos es el segundo flujo en importancia en términos absolutos, pues si a partir de la cifra de que en 2019 llegaron al puerto de San Sebastián de La Gomera 1391116 pasajeros podría estimarse que cada día se mueven de una isla a otra unas 3800 personas, eso significa casi una cuarta parte de la población empadronada en La Gomera. En una cifra similar, unas 3800 personas de una isla a otra, podría estimarse el flujo diario de personas entre Lanzarote y Fuerteventura, a partir de las llegadas de pasajeros a los puertos de Playa Blanca (Lanzarote) y Corralejo (Fuerteventura). Son también importantes los flujos entre Gran Canaria y Fuerteventura, con unas 2700 personas pasando de media de una isla a otra ${ }^{15}$. El resto de flujos entre islas son más difíciles de estimar: así, entre Gran Canaria y Lanzarote (y viceversa) o entre Tenerife y La Palma circulan por vía aérea unas 500 personas diarias, pero los flujos marítimos, ciertamente menores, son más difíciles de estimar.

\section{EL TURISMO DE LAS PERSONAS RESIDENTES DENTRO LAS ISLAS}

Como acabamos de ver, una primera aproximación al fenómeno del estudio de las movilidades en Canarias nos permite ver que, pese a lo que se tiende a pensar debido a la importancia del turismo internacional en Canarias, casi la mitad del movimiento de personas en las islas se da entre las islas, y no entre Canarias y el exterior. Obviamente, buena parte de este movimiento de personas no puede asociarse al «turismo interior en Canarias», porque parte del mismo corresponde tanto a personas residentes de manera permanente en Canarias (que no es exactamente lo mismo que "canarios» ${ }^{16}$ ) que, en muchos casos por motivos no relacionados con el ocio se desplazan entre islas, como a «turistas» que se alojan en una de las islas y que durante un día realizan una excursión a otra. Para estudiar el turismo de las personas

${ }^{15}$ Si sumamos a los más de 300000 pasajeros llegados por vía aérea los más de 665000 que llegaron al puerto de Morro Jable (Fuerteventura), que llegaron en su totalidad desde Gran Canaria, y los 40000 que llegaron a Puerto del Rosario (también en su práctica totalidad desde Gran Canaria), podría estimarse en cerca de un millón de personas al año, es decir, el equivalente a unas 2700 personas diarias por sentido (Gran Canaria-Fuerteventura, o viceversa).

16 Sin pretender entrar aquí en definiciones poco menos que ontológicas acerca de lo que es «ser canario/a», es importante diferenciar entre "personas residentes en Canarias» y "personas nacidas en Canarias», ya que cabe pensar que quizá las personas nacidas en Canarias puedan tener menos movilidad entre islas que las residentes, pero no nacidas en Canarias. Si bien cabe pensar, por ejemplo, que los residentes en La Gomera o Fuerteventura se desplacen a Tenerife o Gran Canaria, ya sea al médico, de compras o a asistir a un evento independientemente de su lugar de nacimiento, las personas residentes en las llamadas islas «menores» nacidas en el extranjero es posible que además tengan que hacerlo para arreglar papeleos en sus consulados. 
residentes en Canarias dentro del archipiélago podríamos utilizar dos enfoques. Por un lado, los datos de la Encuesta de Turismo de Residentes permiten aproximarse al estudio de los canarios(as) como turistas, tanto en el extranjero como en el resto de España y Canarias, ya sea que utilicen alojamientos «turísticos» o que se queden en casas de amigos o familiares.

Con una población de casi 2,2 millones de habitantes (ISTAC 2021a), de los cuales en torno al $60 \%$ realiza al menos un viaje al año, puede estimarse que las personas residentes en Canarias realizan anualmente cerca de seis millones de viajes, que equivalen a unos 28 millones de pernoctaciones (INE 2021c). Es necesario precisar que, a raíz de estas cifras, podría estimarse que 1,3 millones de residentes en Canarias realizan al menos un viaje al año ${ }^{17}$, lo que a simple vista podría parecer contradictorio con la cifra anteriormente mencionada de que, tan solo en Canarias, existen 1,7 millones de «turistas canarios». Esto se debe a que cuando se cuentan turistas (o viajeros) en realidad una misma persona que viaja tres veces contabilizaría como 3 turistas (o viajeros). Para el conjunto de España, y también para otros países, se ha estimado que existe una importante polarización en el consumo turístico, de manera que, grosso modo, podría estimarse que el 20\% de las personas realizan el 80\% de los viajes (Rodríguez González \& Santana Turégano, 2014). Dado que todo hace pensar que un fenómeno similar se da en Canarias, y aunque carecemos de datos acerca de la distribución de los viajes por destino, esto ayuda a comprender por qué es posible que haya casi tantos turistas dentro de Canarias como habitantes en las islas. Dada la gran varianza de la distribución social del consumo turístico, las cifras de que 2 millones de personas realizan cerca de seis millones de viajes al año no hay que interpretarlas como que «cada persona realiza de media tres viajes al año", ya que en realidad lo que puede estar sucediendo, por poner un ejemplo más realista, es que 100000 personas realicen 20 viajes al año (entre las islas o al exterior), que 200000 realicen 10 viajes anuales, otras 20000 realicen 4 viajes, 0,9 millones de personas realicen un viaje anual y que entre estas personas concentren todos los viajes (que implican pernoctación) que se realizan en Canarias, mientas que el $40 \%$ de la población no realizaría viajes. En cualquier caso, casi un $75 \%$ de los viajes y algo más de la mitad de las pernoctaciones que realizan los canarios tienen como destino el propio archipiélago ${ }^{18}$, y en cerca del $40 \%$ de los viajes y las pernoctaciones se utilizan alojamientos de mercado (hoteles, apartamentos), mien-

17 Sería el 60\% de 2,2 millones de habitantes.

${ }_{18}$ De acuerdo a los datos de la «Encuesta de Turismo de Residentes» que realiza el INE. Estas cifras son más elevadas a las de la media espańola: si en Canarias el $80 \%$ de los viajes y el $64 \%$ de las pernoctaciones que se realizaron dentro de Espańa se realizaron dentro del archipiélago, para el conjunto de Espańa más de la mitad de las pernoctaciones y casi la mitad de los viajes se realizaron a otra comunidad autónoma. Y si comparamos los datos con los de otras comunidades, mientras que Cataluña tiene tan solo un $10 \%$ menos de pernoctaciones y viajes dentro de la propia comunidad autónoma que Canarias, en otras como Madrid o Baleares la importancia del viaje a otras comunidades autónomas es significativamente mayor. 
tras que entre un 55 y un $60 \%$ de los viajes se utilizaron casas de amigos y familiares y segundas residencias ${ }^{19}$ (INE 2021d).

Aunque no existen datos desglosados a nivel de comunidad autónoma que ayuden a estimar qué motiva a las personas residentes en Canarias a viajar, para el periodo 2015-2020, para el conjunto de Espańa, en torno al 50\% de los viajes y las pernoctaciones tienen como motivación principal la de «ocio, recreo y vacaciones», en torno al $35 \%$ «visita a amigos y familiares», cerca del 10\% «negocios y otros motivos profesionales» y un 5\% «otros motivos». Por último, respecto a cuándo se realizan los viajes, también para el conjunto de Espańa, casi el $40 \%$ de las pernoctaciones se produjeron durante las vacaciones de verano, mientras que casi un $20 \%$ correspondieron a viajes de fines de semana, en torno a un 5\% cada uno los viajes en Navidad, Semana Santa y puentes y en torno a un $25 \%$ «otros viajes» (INE 2021b) ${ }^{20}$, y cabe pensar que, en la medida en que el comportamiento de los canarios a la hora de viajar viene determinado en gran medida por el calendario laboral, y que este es en gran medida compartido con el resto de los españoles, los datos para Canarias no sean muy distintos de los del total de España. En definitiva, el «retrato robot» de los canarios/as como viajeros sería el de una persona que viaja fundamentalmente por motivos de ocio y recreo, que en gran medida se mueve dentro de su propia comunidad autónoma, que usa establecimientos mercantiles en casi más de la mitad de los casos, algo más que la media de los españoles, y que, tomando como referencia la media de los españoles, tiende a realizar un $40 \%$ de sus pernoctaciones en verano, un $20 \%$ en viajes de fines de Semana, un $15 \%$ en los períodos de Navidad, Semana Santa y puentes y un $25 \%$ en otros períodos.

Ahora bien, otro posible acercamiento al estudio del turismo de los canarios dentro de las islas es el que se puede realizar a través de las Encuestas de Alojamiento Turístico. Este enfoque implica dejar de lado el movimiento de viajeros que implica pernoctación en el que se utilizan como medio de alojamiento segundas residencias o casas de amigos o familiares, aunque es cierto que se trata de un fenómeno que, si bien tiene plena relevancia desde el paradigma de las movilidades, desde el del turismo, y, en general, desde el estudio de la industria turística, tiene poca relevancia: quien reside en una isla y va a visitar familiares a otra posiblemente no es percibido socialmente como «turista», y tampoco se puede considerar como consumidor turístico pues, más allá del transporte, tampoco consume las actividades consideradas típicamente turísticas ${ }^{21}$. Pues bien, si, como suele hacerse habitualmente, nos centramos en lo que suele denominarse «industria turística», es decir, los establecimientos alojativos, y dejamos de lado el turismo que se aloja en establecimientos de no mercado, según datos de Promotur, en el año 2019 se recibieron en los establecimientos turísticos reglados de las islas 1715111 viajeros residentes en Canarias,

19 Lo que el INE denomina «alojamientos de no mercado».

20 Estos datos no se ofrecen desagregados a nivel de comunidad autónoma.

${ }^{21}$ Salvo que se dedique a hacer algunas de las cosas consideradas «típicamente turísticas», como visitar parques temáticos y/o museos. 
de los cuales 637864 residían en Tenerife y 628587 en Gran Canaria (PROMOTUR, 2020). En términos de pernoctaciones, del total de más de 6 millones de pernoctaciones generadas por los residentes en Canarias, 2,39 millones corresponden a residentes en Gran Canaria, y 2,05 a residentes en Tenerife ${ }^{22}$, lo que obedece a que las estancias medias de los grancanarios son mayores que las de los tinerfeños.

Para poder entender lo que significan estos datos en el contexto de la industria hotelera canaria se puede señalar que, de acuerdo a la explotación que realiza el ISTAC de las Encuestas de Alojamiento Turístico, en el período comprendido entre 2009 y $2019^{23}$ en Canarias se realizaron en los establecimientos alojativos del archipiélago entre 91 millones de pernoctaciones (2009) y 116 millones en 2017 (en 2018 fueron 112, y 108 millones en 2019). De todas estas, las pernoctaciones realizadas en Canarias por personas residentes en archipiélago, que están anualmente entre $5 \mathrm{y}$ 6 millones, y representan el 5\% para el total del archipiélago, si bien hay diferencias importantes por islas entre por un lado Fuerteventura, Lanzarote y Tenerife, donde las pernoctaciones realizadas por las residentes en Canarias representan entre el 3 y el $4 \%$ del total de pernoctaciones y Gran Canaria, donde los canarios acumulan en torno al $8 \%$ del total de pernoctaciones ${ }^{24}$ (ISTAC, 2021b). Y para comprender lo que significan estas cifras en el contexto de la industria alojativa española, piénsese, a título de comparación, que de acuerdo a la Encuesta de Ocupación Hotelera para 2019 (INE 2021a) mientras que todos los turistas, de todos los orígenes, realizaron 67 millones de pernoctaciones en los hoteles de Canarias ${ }^{25}$, en los hoteles del País Vasco solo realizaron unos 6 millones, 3,4 en Asturias o 3,2 en Murcia. Es decir, que podría decirse que las personas residentes en Canarias generaron, en el año 2019, tantas pernoctaciones en los apartamentos y hoteles de las islas como todos los turistas, de todas las nacionalidades, en todos los hoteles del País Vasco, o el doble que las que generaron todos los turistas en los hoteles de Murcia o Asturias ${ }^{26}$. en Tenerife.

22 La población oficial para 2019 era de 851231 habitantes en Gran Canaria y 917841

23 No contabilizamos el año 2020 por las peculiaridades asociadas a la pandemia de covid-19.

${ }_{24}$ Obviamente, con fluctuaciones en función del año al que hagamos referencia. En El Hierro el $40 \%$ de las pernoctaciones las realizan personas residentes en Canarias, y en La Gomera y La Palma ronda el 15\%.

25 Y aproximadamente 40 millones más en apartamentos y bungalós.

26 Aunque es necesario señalar que en Murcia buena parte del fenómeno turístico se asocia a las segundas residencias, y en Asturias a establecimientos de turismo rural. 


\section{LOS CANARIOS COMO CLIENTES DE HOTELES Y APARTAMENTOS DE CANARIAS ${ }^{27}$}

El turismo interior o turismo doméstico suele definirse como aquel que se realiza dentro de los límites de una determinada entidad sociopolítica (Pillet Capdepón, 2012), y puede desglosarse entre aquel que utiliza alojamientos de mercado y el que no. Obviamente, dentro del turismo interno cabría hacer subdivisiones en función de las entidades sociopolíticas de referencia. En los territorios continentales, en función de dónde se establezca el límite territorial, a menudo este tipo de movimientos pueden considerarse característicos de lo que se ha dado en llamar «turismo fordista» (Savelli, 1989): en aquellas sociedades en que un porcentaje más elevado de personas viven en ciudades, en climas no siempre agradables y en condiciones de salubridad quizá no ideales, cabría esperar que un porcentaje importante de la población, que dispone de medios para ello, practique lo que suele asociarse al turismo fordista: la escapada de la gris cotidianeidad. Este tipo de razones son las que ayudarían a entender por qué, por ejemplo, muchos ciudadanos del norte de Europa realizan periódicas escapadas hacia lugares de clima cálido, y que en muchos de los países del sur se den periódicos éxodos desde las ciudades hacia los centros costeros, fenómeno que a menudo se asocia a la vuelta a los orígenes cuando los procesos de urbanización son relativamente recientes ${ }^{28}$. En el caso de Canarias cabe pensar que este tipo de turismo se asociaría al «éxodo» que desde las dos grandes conurbaciones capitalinas de Tenerife y Gran Canaria ${ }^{29}$ se produce hacia las playas, tanto de la propia isla como de otras, y que cuando no implica pernoctación puede considerarse excursionismo.

El turismo interior en Canarias puede desglosarse entre el turismo que los habitantes de cada isla realizan dentro de su propia isla y el que realizan en el resto de islas. De acuerdo a los datos de la Encuesta de Alojamiento Turístico, para el período 2009-2020, podría estimarse que unas dos terceras partes, entre un 57\% y un $66 \%$, de las pernoctaciones que realizan las personas residentes en el archipiélago dentro de Canarias corresponden a pernoctaciones realizadas en la propia isla

${ }^{27}$ Como señalamos anteriormente, en realidad habría que decir «las personas residentes en Canarias», pues, por poner un ejemplo, si una persona que nació en el extranjero pero tiene nacionalidad española y reside en Tenerife se desplaza a Gran Canaria y se aloja en un hotel es contabilizado como un «residente en Tenerife que se aloja en Gran Canaria». Por economía del lenguaje usamos esta fórmula, mismo motivo usamos el masculino como genérico en vez de optar por «los/as canarios/as».

28 Sería el caso de las vacaciones en que se vuelve al "pueblo", que en España ya es, en muchas ocasiones, no el pueblo propio, sino el de los padres y/o abuelos.

${ }^{29}$ De acuerdo a la explotación estadística del Padrón, la zona metropolitana de Tenerife, incluyendo a los municipios de Santa Cruz de Tenerife, San Cristóbal de La Laguna, Tegueste y El Rosario, con 396888 habitantes, concentra al 18\% de la población de Canarias, mientras que la de la Gran Canaria, con 540 742, incluyendo a los municipios de Las Palmas de Gran Canaria, Telde, Arucas y Santa Brígida, concentra al 25\% de la población del archipiélago. La concentración respecto de la propia isla es aún mayor: la zona metropolitana capitalina concentra al $63 \%$ de la población en Gran Canaria y al $42 \%$ en Tenerife. 
de residencia. Si analizamos las islas como mercados emisores, estudiando el conjunto de las pernoctaciones que los residentes de todas las islas realizan en todo el archipiélago Gran Canaria es el principal mercado emisor, acumulando cerca de la mitad de las pernoctaciones para todo el período 2009-2020, seguida de Tenerife, que concentra en torno a un 30\%, Fuerteventura y Lanzarote, que concentran en torno al 8\%, La Palma aproximadamente el 6\% y La Gomera y El Hierro en torno al 3\%. De acuerdo a las teorías sobre el turismo "fordista" mencionadas anteriormente, esto puede interpretarse como consecuencia de que, si dicho tipo de turismo se relaciona con el grado de urbanización, es la isla en que se concentra más población en un gran área metropolitana aquella en que más se da este tipo de turismo ${ }^{30}$. De todas formas, el perfil de los canarios como turistas emisores (siempre dentro de Canarias) es relativamente distinto para cada isla, lo que seguramente obedece a distintas tipologías de turismo.

El análisis de las pernoctaciones de las personas residentes en Canarias en establecimientos turísticos registrados del archipiélago, en el período 2009-2020, para el que el ISTAC provee información, permite un acercamiento al comportamiento de los canarios cuando hacen turismo en Canarias. Es necesario precisar, en cualquier caso, que se analizan solo los casos en que se alojan en establecimientos reglados, quedando fuera de este análisis, por lo tanto, el alojamiento en segundas residencias o casas de amigos o familiares. Pues bien, desglosando el análisis por islas, y comenzando por Lanzarote, más de la mitad de las pernoctaciones realizadas por los lanzaroteños en hoteles y apartamentos de Canarias se realizan en la propia isla. Teniendo en cuenta la distribución de la población en la isla y su variabilidad climática y paisajística, cabe pensar que se trata, sobre todo, de lo que podría considerarse un turismo "vacacional»: personas que pasan una o varias noches en hoteles o apartamentos de la isla (por lo que casi podría considerarse que en su «entorno habitual») $)^{31}$, por motivos que seguramente tienen que ver con el disfrute de lo que estos tienen que ofrecer (básicamente, sol y playa) en un entorno social (con amigos y familiares). Distinto sería el caso de las pernoctaciones de los lanzaroteños en Gran Canaria (el $22,7 \%$ del total), que, en la medida en que en dicha isla está la capital de la provincia a la que pertenece Lanzarote, se pueden asociar a un «turismo» más heterogéneo: desde las compras o la asistencia a eventos al turismo urbano y otro tipo de actividades que o bien no se pueden realizar en la isla de origen o al menos

\footnotetext{
${ }^{30}$ Aun cuando para el conjunto de la isla la población de Tenerife sea algo mayor que la de Gran Canaria.

31 En total, para el período considerado, cerca de 5 millones de pernoctaciones, algo menos de 500000 anuales; recordemos que Lanzarote tenía en 2020 una población de 155182 habitantes. Más de la mitad de la población se concentra en su capital, Arrecife, y el 82\% en una conurbación que además de este municipio y al de San Bartolomé incluye dos de los principales destinos insulares, Tías (Puerto del Carmen) y Teguise (Costa Teguise). Un habitante del lugar más alejado de la capital no necesita pernoctar si ha de acudir al médico y/o realizar gestiones en la misma. En realidad, cabe pensar que buena parte de este casi medio millón de pernoctaciones que realizan los lanzaroteños en Lanzarote las hace en Tías, Teguise y Yaiza personas que viven en la conurbación capitalina, por lo que difícilmente puede decirse que salgan de su «entorno habitual».
} 
pueden realizarse mejor en la isla de destino ${ }^{32}$. Por último, y en lo que hace referencia al resto de islas, un $14 \%$ de las pernoctaciones de los lanzaroteños dentro de Canarias se realizan en Fuerteventura, un 7\% en Tenerife y un 2\% en La Palma (La Gomera y El Hierro no llegan al 1\% en su conjunto). En cuanto a Fuerteventura, las pautas de consumo de servicios de alojamiento no son muy distintas: entre 2009 y 2020 los residentes en la isla realizaron 3,3 millones de pernoctaciones en Canarias, el 55\% de ellas en la propia isla majorera, un 26\% en Gran Canaria, un $10 \%$ en Lanzarote, un 6\% en un 2\% entre La Palma, La Gomera y El Hierro.

En cuanto a Gran Canaria, la isla que más peso tiene en el turismo interior en Canarias, el 68\% de las pernoctaciones de los residentes en la isla se realizan en la propia isla, y aquí sucede algo similar a lo anteriormente comentado para el caso de Lanzarote: teniendo en cuenta la distribución de la oferta alojativa ${ }^{33}$ y la de la población ${ }^{34}$, buena parte de las pernoctaciones se realizan en lugares que pueden estar a media hora de viaje en coche del lugar de residencia, por lo que resulta también difícil hablar de que, en estos casos, el turismo implique salir de "entorno habitual». El resto de las pernoctaciones de los grancanarios en Canarias se reparten entre un 13,7\% en Fuerteventura, un 8,5\% en Tenerife, un 6,7\% en Lanzarote y un $2 \%$ en La Palma (La Gomera y El Hierro juntos no llegan al 1\%). Aunque el caso de las pernoctaciones de los grancanarios en Tenerife puede obedecer a pautas un poco más variadas, alejadas del turismo de sol y playa (visitas, eventos, turismo cultural, negocios), parece que, en términos generales, cuando los canarios practican turismo dentro de las islas siguen las pautas de lo que tradicionalmente se ha considerado «turismo fordista». Al fin y al cabo, cuando los canarios deciden pagar por alojarse en un hotel o apartamento de las islas es para ir a la playa, ya sea en la propia isla o bien en islas, como Fuerteventura y Lanzarote, que lo que ofrecen es fundamentalmente playa.

Y esta misma idea se repite si analizamos la distribución de las pernoctaciones en Canarias de los residentes en Tenerife: si bien el porcentaje de pernoctaciones que se realizan en la propia isla sigue la tónica general de ser mayoritario $(57 \%)$ la siguiente isla en importancia es Gran Canaria, que acumula un 14,6\% de las pernoctaciones de los tinerfeńos en Canarias; le siguen La Gomera y La Palma (8,9\% y 8,4\%, respectivamente), seguidas de Lanzarote (5\%) Fuerteventura y El Hierro $(1,7 \%)$. En cuando a las islas más occidentales, que por su menor población son menos importantes en términos cuantitativos, La Gomera y La Palma siguen una

32 Es necesario recordar, una vez más, que no estamos aquí contabilizando a residentes en Lanzarote (quizá nacidos fuera de la isla) que visitan Gran Canaria y se alojan en casas de amigos o familiares.

${ }^{33}$ Concentrada en los municipios de San Bartolomé de Tirajana, Mogán y Las Palmas de Gran Canaria.

${ }^{34}$ Además de las 540000 personas que viven en el área metropolitana, como vimos anteriormente, un total de 760000 , el $88 \%$ de la población de la isla, vive entre la zona metropolitana y los municipios del este y sur de la isla (Mogán, San Bartolomé de Tirajana, Santa Lucía de Tiraja, Agüimes e Ingenio). 
pauta similar: un $20 \%$ son pernoctaciones en la propia isla, un $40-50 \%$ en Tenerife y un 20\% en Gran Canaria. Por último, El Hierro presenta una pauta distinta, pues apenas hay pernoctaciones dentro de la propia isla, y la mayoría (el 70\%) de las pernoctaciones que hacen los herreños en Canarias se concentran en Tenerife, quedando un 10\% en Gran Canaria y otro 10\% entre Fuerteventura y Lanzarote.

En cualquier caso, dado el distinto peso demográfico de cada isla sobre el total de Canarias, al agregar los datos podríamos decir que, de los más de 63 millones de pernoctaciones que realizaron los canarios en establecimientos turísticos reglados del archipiélago en el período 2019, a partir de la explotación de los datos facilitados por el ISTAC podría decirse que el 32,6\%, del total, es decir, más de 20 millones, correspondieron a pernoctaciones de residentes en Gran Canaria en la propia isla de residencia. Unos 11 millones, el 18,5\% del total, corresponden a residentes en Tenerife que realizaron pernoctaciones en la misma isla; los residentes en Gran Canaria realizaron 9,7 millones de pernoctaciones en otras islas (15,4\% del total), mientras que los residentes en Tenerife realizaron 8,5 millones de pernoctaciones en otras islas (13,5\% del total). En conjunto, por lo tanto, tinerfeños y grancanarios realizaron el $88 \%$ de las pernoctaciones que realizaron los canarios en las islas en el período 2009-2020, lo que puede parecer lógico, si se tiene en cuenta que ambas islas representan aproximadamente ese porcentaje de la población total del archipiélago. Sin embargo, las personas residentes en Gran Canaria, con un $48 \%$ del total, tienen un peso significativamente mayor al que les correspondería por población.

¿Por qué realizan más pernoctaciones los residentes en Gran Canaria que los residentes en Tenerife ${ }^{35}$. Una posible razón podría ser, como se ha sugerido anteriormente, la concentración territorial. En el marco de un turismo fordista asociado al «escape» de las condiciones de vida en la urbe, pese a que en su conjunto Tenerife tenga una población algo mayor a la de Gran Canaria, al estar más poblada la zona metropolitana de Las Palmas que la de Tenerife ello generaría mayor volumen de ese turismo de «escape». Aunque, por otro lado, también es cierto que esta mayor dispersión de la población y el hecho de que las distancias en Tenerife sean mayores podrían generar un cierto tipo de turismo «urbano» que no llega a compensar lo anterior ${ }^{36}$. Otra posible razón tiene que ver con las diferentes características de la oferta alojativa de cada isla, con mayor peso del sector extrahotelero en Gran Canaria. La hipótesis en este caso sería que los apartamentos y bungalós son un tipo de

35 No es que los residentes en Gran Canaria realicen más pernoctaciones dentro de su propia isla mientras que los tinerfeños vayan más a otras islas; aunque año a año las cifras pueden variar, de media los residentes en Gran Canaria realizan tantas o más pernoctaciones en otras islas distintas de la propia que las que realizan los residentes en Tenerife en otras islas.

${ }^{36}$ Es decir, entre el suroeste y el noroeste de Tenerife existe una población de cerca de 300000 habitantes que está a una distancia de más de una hora en coche de la capital y que, por lo tanto, podría, en caso de desplazarse a la capital, usar los servicios de un hotel para alojarse (también es posible que cuenten con segunda residencia). Por el contrario, la distribución de la población y los sistemas de transporte existentes hacen que menos de 60000 personas en Gran Canaria vivan lo suficientemente alejadas de la capital como para hacer recomendable alojarse cuando han de acudir allí por cualquier motivo. 
oferta que se adapta más a los requerimientos de los residentes en Gran Canaria a la hora de hacer turismo interno, y como en Gran Canaria es más abundante tiene más importancia en dicha isla. En Tenerife, pese a que sea isla con mayor oferta alojativa, bien sea por los canales de comercialización, o porque esta no resulta tan atractiva a la población residente en Canarias, el peso del turismo interno es menor. Desglosando los datos de las Encuestas de Oferta Alojativa del ISTAC se puede calcular que para el período 2009-2020 unas dos terceras partes (entre el 60 y el 69\%) de las pernoctaciones que realizan los canarios en Gran Canaria se hacen en apartamentos y bungalós, porcentaje que baja hasta aproximadamente una tercera parte (entre el 28 y el 39\%) en Tenerife.

Una tercera hipótesis que permitiría comprender por qué es más importante el turismo interno en Gran Canaria que en Tenerife tiene que una cultura de consumo relativamente distinta ${ }^{37}$, si bien la contrastación de esta hipótesis excedería con mucho el ámbito de este trabajo. Incidiendo en la idea de la diferenciación (Santana Turégano, 2000) entre el turismo y otras formas de ocio, la idea sería que, mientras que en Tenerife pueden estar más generalizadas otras formas de ocio ${ }^{38}$, el «ir al sur de apartamento» es una forma de ocio que, hasta cierto punto, estaría más generalizada en la cultura de consumo en Gran Canaria. Y esto se explicaría por los procesos mediante los cuales se acabó construyendo la actual oferta alojativa. Tradicionalmente en Gran Canaria buena parte de la oferta de alojamiento se centraba en apartamentos y bungalós, que eran propiedad de pequeños ahorradores locales, que los cedían para la explotación turística y que los disfrutaban en verano, aprovechando la bajada en la demanda de turismo internacional hacia Canarias en esos meses (Santana Turégano, 2003; Santana Turégano, 2005). Tras más de medio siglo de desarrollo turístico, muchos miembros de las clases medias grancanarias, que de nińos pasaban buena parte del verano en apartamentos del sur, y ahora son adultos, presentan una importante propensión a consumir ese producto («vacaciones en el sur»), por haber sido socializados en ese tipo de prácticas ${ }^{39}$. Dado que este tipo de procesos tuvieron menos importancia en Tenerife, donde la oferta alojativa es en mayor medida hotelera, y en mayor parte controlada por cadenas cuyos centros de decisiones están fuera, es posible que la población local no consiga encontrar ofertas lo suficientemente atractivas como para consumir «turismo local $»^{40}$. Para terminar

${ }^{37}$ En realidad, al no existir datos desglosados a nivel insular, no podemos saber si la mayor propensión a consumir alojamientos turísticos en Gran Canaria que en Tenerife se traslada en una mayor propensión en general hacia el consumo turístico en la primera isla que en la segunda.

${ }^{38}$ Como el ir de restaurante.

39 Además, hay que tener en cuenta que ya habían sido socializados en una especie de «Time Sharing»: si de niño tus padres tenían un apartamento en el complejo Roque Bentayga (nombre ficticio), no quiere eso decir que en el mes de julio (o agosto) te quedaras en «tu apartamento», sino en uno que estuviera disponible, que podía no ser el propio, lo que quizá facilite que cuando llegues a la edad adulta no veas tan extraño el pagar por alojarte en un apartamento, aunque esté en un lugar al que podrías ir y volver cada día desde tu propia residencia

${ }^{40}$ Expresado en forma coloquial, una cosa sería pagar 50 euros/noche por un apartamento en el sur en el que poder meter a toda la familia y no tener que estar yendo y viniendo cada día a la 
con el análisis del turismo de los canarios dentro de Canarias es importante señalar que en el año 2018 se produjo un cambio en los descuentos al transporte de las personas residentes, lo que ha abaratado significativamente el coste del viaje entre las islas. Esto se ha visto reflejado en un incremento en un 15\% de las pernoctaciones en islas distintas de la propia entre 2017 (con el descuento antiguo) y 2019 (con el descuento nuevo), sin que hayan disminuido las pernoctaciones en la propia isla (PROMOTUR, 2020). Obviamente, el impacto de la pandemia de covid-19 en 2020 impide que los datos de los años 2020 y 2021 sean comparables.

\section{CONCLUSIONES}

El objetivo inicial de este trabajo era aproximarse al estudio del turismo interior de los canarios. Plantearse esta cuestión como objeto de estudio nos ha llevado a la necesidad de adaptar un enfoque más cercano a lo que Urry considera el paradigma de las movilidades que al del turismo, pues, de hecho, dada la importancia del turismo internacional en Canarias muchos de los fenómenos asociados al turismo interno, y a la movilidad de las personas no directamente asociadas al turismo, resultan difícilmente visibles. Los estereotipos propios del sentido común nos llevan a pensar que los canarios no son turistas, que no son turistas en Canarias, cuando lo cierto es que cerca de un 7\% de todas las pernoctaciones en los establecimientos alojativos de Canarias las realizan personas residentes en las islas. Y para poner las cifras en contexto conviene recordar que, por ejemplo, en Gran Canaria generan casi tantas pernoctaciones los canarios como los suecos (véase ISTAC 2021b). Y que los canarios en Canarias realizan tantas pernoctaciones como todo el conjunto de los turistas en el País Vasco (INE, 2021b; ISTAC, 2021b).

Los resultados del trabajo realizado han permitido, en primer lugar, dimensionar la importancia del turismo interior en Canarias, entendido este como "pernoctaciones realizadas por residentes en Canarias establecimientos turísticos reglados del archipiélago", y ponerlo en el contexto de las movilidades que tienen lugar en el archipiélago. A partir del trabajo realizado podría estimarse que, para el período 2009-2019 (el 2020 presenta resultados atípicos debido a la pandemia de la covid-19) los habitantes en las islas realizan anualmente, de media, unos 6 millones de pernoctaciones en establecimientos turísticos reglados del archipiélago. Estas cifras, que representan cerca del 3\% del total de pernoctaciones en las islas de Fuerteventura, Lanzarote y Tenerife, y del 7-8\% en Gran Canaria, se realizan, en sus dos terceras partes, dentro de la propia isla de residencia, y en mayor medida en la isla de Gran Canaria. Es decir, al menos en lo que hace referencia al turismo que sea realiza en

playa (Gran Canaria) y otra bastante distinta, por ahorrarte subir y bajar al sur, pagar 100 euros por una habitación en el que solo cabe una pareja (Tenerife). Por otro lado, si el que gestiona las reservas del apartamento es un conocido, y sabe que lo tiene vacío y sabe que te interesa, te ofrezca quizá un buen precio (Gran Canaria). Si las reservas del hotel las llevan desde la central de la cadena en Baleares, va a ser más difícil que la población local acceda a buenos precios. 
el interior de las islas, parece que el turismo se mueve aún bastante en el marco de lo que se denominaba «turismo fordista».

En un contexto turístico mundial marcado por la pandemia de la covid-19, y en el que la estrategia institucional parece ir hacia el desarrollo de lo que se ha dado en llamar "nómadas digitales» ${ }^{41}$, una forma de movilidad que difícilmente puede considerarse turística, parece necesario repensar las herramientas conceptuales con las que nos enfrentamos al estudio de los procesos tradicionalmente considerados como «turismo». Como ya señalaran, entre otros, Álvarez Souza et al., 2019, algunas de las ideas implícitas en el concepto de turismo, con la idea de "tour», de un ir y volver de un sitio a otro, parecen cada vez más difusas. Así, en unas islas en las que más del $20 \%$ de la población ha nacido fuera, ¿cómo diferenciar entre cuándo se va y cuándo se vuelve? En la actualidad, muchas personas que tienen su origen en, por ejemplo, el Reino Unido, Alemania o Suecia, pero que residen en Canarias, y acuden al menos una vez al año a visitar a sus familiares, podrían ser considerados como turistas «Visiting Friends and Relatives», pero son turistas canarios en los países anteriormente citados, y no viceversa. Por otro lado, la necesidad de repensar la diferenciación entre el turismo y otras formas de ocio, que ya hace más de 20 años que se señalaba (Santana Turégano, 2000), se hace ahora más necesaria que nunca. Quizá no vivamos una modernidad completamente líquida (Bauman, 2015), en la que ya no aspiremos a una casita en la que envejecer, sino a una vida en constante búsqueda de nuevos destinos, pero es cierto que las fronteras entre lo cotidiano y lo extraordinario, entre el ocio y el negocio, entre lo local y lo global, se han transformado por completo. Hasta el punto de que, en lo que a veces es considerado la primera región turística de Europa (Kotzeva et al., 2019), el consumo "turístico» de los locales es, en muchas ocasiones, también local. Lo cual, por otra parte, podría parecer elección perfecta de un consumidor perfectamente racional: si lo que quiero es descansar, y millones de europeos vienen cada año aquí a descansar, ¿para qué irse fuera? En cualquier caso, el trabajo realizado también ha permitido encontrar lo que se podrían considerar «diferencias microculturales» en la propensión a consumir servicios turísticos, lo que refuerza la importancia de hacer un estudio de los fenómenos socioeconómicos enraizado en la historia. En un contexto en el que el Big Data parece ser la gran promesa para mejorar el conocimiento en un montón de campos, lo que hemos hecho en este trabajo no ha sido más que un trabajo artesano, muy primitivo en términos tecnológicos y matemáticos, pero informado desde un punto de vista teórico. Al final, aunque estemos utilizando información cuantitativa producida por otros medios, es necesario un conocimiento teórico que guíe tanto la recogida como el análisis de la información.

Por supuesto, el trabajo realizado tiene implicaciones prácticas y líneas a continuar desarrollando. En el contexto del turismo postcovid, y dado que, pese a la importancia del turismo en Canarias, prácticamente no se han hecho estudios sobre

\footnotetext{
${ }^{41}$ Véase, por ejemplo, https://www.europapress.es/islas-canarias/noticia-canarias-lanza-campana-captar-segmento-silver-nomadas-digitales-20200714161332.html.
} 
el turismo interior (una excepción sería Martínez González et al., 2017), parece necesario profundizar en el conocimiento de las personas que residen en Canarias como consumidoras de servicios considerados típicamente turísticos. Lo que parece ser la estrategia actual de muchas regiones tradicionalmente turísticas de reconvertirse hacia polos de atracción de los llamados «nómadas digitales» implica importantes cambios. Sin ir más lejos, y, por obvio que pueda resultar, parece que a veces no se tenga presente, una habitación de hotel puede ser un entorno adecuado para unas vacaciones de una semana, pero puede no serlo para una estancia de varios meses. Claro que otra cuestión es que, una vez asentados en una isla, los nómadas digitales realicen turismo interno dentro de las islas, y una persona que, por ejemplo, se haya asentado en La Gomera o Fuerteventura visite Tenerife o Gran Canaria. El estudio de los fenómenos tradicionalmente asociados al turismo desde un punto de vista un poco más amplio, como es el paradigma de las movilidades, puede ayudar a comprender mejor este tipo de fenómenos. Esperamos haber contribuido a dar un primer paso con este. 


\section{BIBLIOGRAFÍA}

Álvarez Sousa, A., Mantecón, A. y Puertas-Cañaveral, I. (2019). Introducción: Turismo y modernidad, en sociología del turismo (1. ${ }^{a}$ ed.). Madrd: Centro de Investigaciones Sociológicas.

Aranda Palmero, E. (2001). «Estadística de movimientos turísticos de los españoles (FAMILITUR)». Estudios Turisticos, (148), 47-67.

Bauman, Z. (2015). Modernidad líquida. Fondo de cultura económica.

Bianchi, R. (2017). En Harrison D. y Sharpley R. (eds.), The political economy of mass tourism and its contradictions. CAB International.

Bianchi, R. (2018). «The political economy of tourism development: A critical review». Annals of Tourism Research, 70, 88-102. https://10.1016/j.annals.2017.08.005.

Callon, M. (2010). «Performativity, misfires and politics». Journal of Cultural Economy, 3(2), 163-169.

CAmic, C. (1987). «The making of a method: A historical reinterpretation of the early parsons». American Sociological Review, 421-439.

Díaz, P., Santana, A., Parra, E. y Calero, F.J. (2017). «Laboratorio atlántico de turismo: Competitividad, innovación y gobernanza turística en canarias». Ambienta, 118, 58-65.

González Martín, E. y Velasco Gimeno, M. del C. (2021). «Encuestas de ocupación en alojamientos turísticos. índices de precios e indicadores de rentabilidad del sector hotelero». Indice: Revista de Estadistica y Sociedad, (80), 26-32.

Hernández Martín, R. (ed.) (2011). La cuenta satélite del turismo de canarias. metodología. metodología, resultados y reflexiones. Fundación FYDE-CajaCanarias.

INE (2021a). Encuesta de ocupación hotelera 2019: Resultados por comunidades y ciudades autónomas-pernoctaciones de los viajeros por comunidades y ciudades autónomas, país de residencia y meses. https://www.ine.es/jaxi/Datos.htm?tpx=37147.

INE (2021b). Encuesta de turismo de residentes: Resultados nacionales: Viajes, pernoctaciones, duración media y gasto. https://www.ine.es/dynt3/inebase/es/index.htm?padre=3811\&capsel=3812.

INE (2021c). Encuesta de turismo de residentes. resultados. https://www.ine.es/dyngs/INEbase/ es/operacion.htm?c=Estadistica_C\&cid $=1254736176990 \&$ menu $=$ resultados $\&$ i$\mathrm{dp}=1254735576863 \#$ !tabs-1254736195601.

INE (2021d). Resultados por comunidad autónoma de residencia: Viajes, pernoctaciones, duración media y gasto por tipo de alojamiento principal, según comunidad autónoma de residencia de los viajeros. https://www.ine.es/jaxiT3/Datos.htm?t=24943.

ISTAC (2021a). Cifras oficiales de población. http://www.gobiernodecanarias.org/istac/estadisticas/ demografia/poblacion/cifraspadronales/E30245A.html.

ISTAC (2021b). Encuestas de alojamiento turístico/series mensuales. canarias por islas y municipios turísticos. 2009-2020: Pernoctaciones, viajeros entrados y viajeros alojados según lugares de residencia por islas de alojamiento de canarias y periodos. http://www.gobiernodecanarias. org/istac/jaxi-istac/menu.do?uripub=urn:uuid:40dab52d-bc0e-4986-969c-05d6b9260959.

ISTAC (2021c). Estadística de movimientos turísticos en fronteras de canarias. http://www.gobiernodecanarias.org/istac/estadisticas/sectorservicios/hosteleriayturismo/demanda/E16028B. html. 
ISTAC (2021d). Hostelería y turismo: Demanda turística: Turistas y pasajeros. http://www.gobiernodecanarias.org/istac/estadisticas/sectorservicios/hosteleriayturismo/.

Kotzeva, M., Brandmüller, T. y Önnerfors, Å. (2019). Eurostat regional yearbook: 2019 edition (2019 edition ed.). Luxembourg: Publications Office. https://10.2785/411990.

Kotzeva, M., Brandmüller, T., Önnerfors, Å. y ReInecke, P. (2018). Eurostat regionalyearbook: 2018 edition (2018 edition ed.). Luxembourg: Publications Office. https://10.2785/220518.

Martínez González, J.A., Parra-Lopez, E. y Buhalis, D. (2017). «The loyalty of young residents in an island destination: An integrated model». Journal of Destination Marketing \& Management, 6(4), 444-455. https://10.1016/j.jdmm.2016.07.003.

OMT/WTO (2018). The UNWTO world tourism barometer. The Tourism Market Trends Programme of UNWTO.

Pillet Capdepón, F. (2012). «El turismo de interior en la españa peninsular: El patrimonio territorial como destino turístico». Boletín De La Asociación De Geógrafos Españoles (59). https://10.21138/bage.1461.

PROMOTUR (2020). Estadísticas de turismo interno. 2019. https://turismodeislascanarias.com/ sites/default/files/promotur_turismo_interno_2019.pdf.

Rodríguez González, P. y Santana Turégano, M.Á. (2014). «Consumo turístico y desigualdad social en españa». Pasos (Tenerife-Canary Islands), 12(1), 29-51. https://10.25145/j. pasos.2014.12.003.

Santana Turégano, M.Á (ed.). (2000). Turismo fordista y posturismo. una primera aproximación a la operativización de conceptos. Proyecto Sur de Ediciones.

Santana Turégano, M.Á. (2003). En Martin, A. (ed.), Formas de desarrollo turístico, redes y situación de empleo: El caso de maspalomas (gran canaria). Bellaterra Universitat Autònoma de Barcelona.

Santana Turégano, M.Á. (2005). «Turismo, empleo y desarrollo». Papers: Revista De Sociologia, (77).

Savelli, A. (1989). Sociología del turismo. Milano: Franco Angeli.

Sheller, M. y Urry, J. (2006). The new mobilities paradigm. Environment and Planning A, 38(2), 207-226.

Stark, D. (2009). The sense of dissonance: Accounts of worth in economic life. Princeton University Press.

URRY, J. (2012). Sociology beyond societies: Mobilities for the twenty-first century. Routledge.

URRY, J. (2016). Mobilities: New perspectives on transport and society. Routledge. 